\title{
e-Phaïstos
}

e-Phaïstos

Revue d'histoire des techniques / Journal of the history

of technology

IX-1 | 2021

Autour de Léonard de Vinci

\section{Navigations et installations lacustres sur les Hautes Terres du Mexique : les cas mexica et tarasque}

Navigation and lake installations in the Mexican highlands: the Mexica and

Tarascan cases

\section{Alexandra Biar}

\section{(2) OpenEdition}

Journals

Édition électronique

URL : https://journals.openedition.org/ephaistos/8441

DOI : 10.4000/ephaistos.8441

ISSN : 2552-0741

Éditeur

IHMC - Institut d'histoire moderne et contemporaine (UMR 8066)

Référence électronique

Alexandra Biar, « Navigations et installations lacustres sur les Hautes Terres du Mexique : les cas mexica et tarasque », e-Phaïstos [En ligne], IX-1 | 2021, mis en ligne le 28 avril 2021, consulté le 18 septembre 2021. URL : http://journals.openedition.org/ephaistos/8441 ; DOI : https://doi.org/ 10.4000/ephaistos.8441

Ce document a été généré automatiquement le 18 septembre 2021.

Tous droits réservés 


\section{Navigations et installations} lacustres sur les Hautes Terres du Mexique : les cas mexica et tarasque

Navigation and lake installations in the Mexican highlands: the Mexica and Tarascan cases

\section{Alexandra Biar}

Thèse : références bibliographiques

Alexandra BIAR, Navigation et installations lacustres sur les hautes terres mexicaines : les cas mexica et tarasque, Thèse de doctorat en Archéologie des Amériques, Université Paris 1 Panthéon-Sorbonne, Paris (France), soutenue le 24 novembre 2017, un volume de texte (507 p.).

\section{Directeurs de thèse}

FAUGÈRE, Brigitte, Professeur des Universités, Université Paris 1 PanthéonSorbonne

LÓPEZ LUJÁN, Leonardo, Directeur de recherche, INAH (Mexique)

\section{Jury :}

TALADOIRE, Éric, Professeur émérite, Université Paris 1 Panthéon-Sorbonne RIETH, Éric, Directeur de recherche, CNRS

HAMMOND, Norman, Professeur émérite, Boston University

BÉRARD, Benoît, Maître de conférences HDR, Université des Antilles et de la Guyane

1 Monsieur le Président, chers membres du jury, chers auditeurs, c'est un plaisir d'être ici devant vous aujourd'hui pour soutenir ma thèse de doctorat à laquelle vous avez tous apportés votre expertise, vos conseils et votre soutien. Nous allons tous embarquer dans quelques instants sur les Hautes Terres du Mexique où, un peu plus d'un siècle avant l'arrivée des Conquistadors, deux civilisations contemporaines et ennemies ont 
réussi à bâtir leurs empires au contact d'un milieu lacustre dont chacun, à sa manière, a su tirer parti. Mais avant de vous exposer la méthodologie, les résultats et les conclusions apportés par ce travail de recherche, regardons ensemble, en guise d'introduction, cette courte vidéo ${ }^{1}$.

Bien qu'aujourd'hui la navigation Maya soit la source d'un évènement sportif et culturel de grande envergure, elle a été considérée, dès les années 1970, comme un sujet de recherche de premier ordre par des archéologues comme Norman Hammond ici présent. Leurs travaux liés à l'étude de la navigation maritime Maya à travers des sources exclusivement iconographiques, se sont principalement concentrés le long des côtes de la Péninsule du Yucatán, mais qu'en est-il de la navigation dans les eaux intérieures? Celle sur les lacs et les rivières? Pour ce faire, il aura fallu attendre les années 2010 et l'arrivée d'une vague de jeunes chercheurs pour travailler ces sujets avec la même rigueur scientifique. Nous citerons les travaux de Nicolas Lira au sud du Chili, ceux de Christophe Delaere sur la partie bolivienne du Lac Titicaca et, enfin, au Mexique, ceux de Mariana Favila dans les lagunes côtières de l'État de Veracruz et le nôtre sur les lacs des Hautes Terres. Ces recherches témoignent d'une dynamique nouvelle qui a trop longtemps fait défaut. En effet, en raison d'une vision eurocentrée sur la partie terrestre de l'archéologie, nous avons trop souvent fait des eaux intérieures plutôt des toiles de fonds que des objets d'étude systématique.

Dans une aire culturelle où la géographie conspire contre la fluidité des échanges, les sociétés mésoaméricaines comme les mexicas et les tarasques ont su trouver des réponses techniques adaptées à leurs besoins. À une époque où l'acheminement de marchandises et de biens s'effectue principalement à dos d'homme, ces deux civilisations ont appris à contrôler leur environnement, c'est-à-dire un milieu aquatique mythique : les lacs. Nos recherches se sont donc intéressées à la pratique de la navigation lacustre et aux installations spécifiques qui lui sont associées.

De par la nécessité d'une approche transversale, ce sujet se positionne dans un cadre pluridisciplinaire, entremêlant archéologie, ethnohistoire et ethnoarchéologie. Premièrement, l'archéologie nous donne accès à des données liées aux questions d'urbanisme et de distribution spatiale des installations comme les ports ou les embarcadères. Deuxièmement, les sources ethnohistoriques regroupent à la fois les témoignages écrits des premiers Conquistadors comme ceux de Cortès ou du frère Sahagun, des cartes anciennes comme celles dites d'Uppsala ou de Nuremberg, des sources iconographiques à la fois réalisées au lendemain de la Conquête par des artistes indigènes, mais aussi des photographies prises à l'aube du $\mathrm{XX}^{\mathrm{e}}$ siècle. Troisièmement, l'ethnoarchéologie, à travers nos nombreux séjours sur le terrain, nous a permis de recueillir des témoignages des descendants de ces navigateurs du Nouveau Monde. Ceux-ci ont été précieux tant sur la manière d'appréhender le paysage culturel mais aussi les questions techniques liées à la navigation. Ainsi, l'objectif premier de ce travail consiste à délimiter le cadre d'un nouveau champ de recherche à partir d'une étude analytique et systématique d'un corpus de données éclectiques, autour de l'exploitation d'un mode de transport aquatique. 


\section{Environnement lacustre et installations humaines sur les Hautes Terres}

5 En Mésoamérique, c'est dans les hautes terres mexicaines que nous dénombrons la plus grande concentration de systèmes lacustres. Toutefois, seuls les lacs des Bassins de Mexico et de Pátzcuaro ont été convertis en de véritables centres politiques, économiques et culturels à l'origine de l'émergence de l'Empire mexica et du Royaume tarasque à la période Postclassique (1350-1521). Toutefois, ces deux régions ont connu une évolution très différente. Aujourd'hui, les lacs du Bassin de Mexico ont été ensevelis sous la mégalopole de Mexico. Quelques réminiscences de son passé aquatique subsistent dans la zone touristique de Xochimilco. Ceci explique que l'accès aux données archéologiques soit extrêmement complexe et tributaire des travaux d'urbanisation qui, seuls, donnent accès à de nouvelles découvertes. Á l'inverse, le lac de Pátzcuaro est toujours navigable et partiellement exploité par quelques embarcations dirigées par de vieux pêcheurs avec lesquels nous avons partagés au cours de nos séjours insulaires.

6 Au-delà de leur évolution, ces deux bassins ont formé l'écrin de deux capitales qui se différencient par leur localisation. D'un côté, il y a Tenochtitlan, la capitale des Mexica, fondée en 1325 sur un îlot situé au milieu d'un lac d'eau saumâtre. Accessible uniquement par voie d'eau à ses débuts, elle va rapidement être reliée aux rives du lac par de grandes chaussées, pour en faciliter l'accès par voie terrestre. Toutefois sa situation insulaire en a fait la victime des fluctuations du niveau des eaux des lacs qui ont causé plusieurs inondations ravageuses. Dès lors, de grands travaux hydrauliques tels que les chaussées et des digues vont être construits sur les eaux du lac pour tenter de la protéger tout en régulant le niveau des eaux. Cortès, en la découvrant, parlera de la Venise du Nouveau Monde. D'un autre côté, Tzintzuntzan, la capitale des Tarasques, s'élevait sur les hauteurs des rives du lac de Pátzcuaro. Fondée elle-aussi en 1325, elle était accessible par voie navigable et terrestre. Sa hauteur lui permettait d'avoir une vue d'ensemble du lac, de ses îles et du trafic de pirogues qui desservait les autres centres riverains. Ce sont donc leurs situations, insulaire ou riveraine, qui vont être l'élément clef dans l'appréhension du paysage culturel lacustre.

\section{Paysage culturel lacustre, navigation et aménagements lacustres}

7 Emprunté au concept du paysage culturel maritime définit par Westerwald, nous proposons dans ce travail celui de paysage culturel lacustre, basé sur les mêmes fondamentaux qui combinent des ouvrages de l'homme et de la nature. Nous pourrons parler d'une approche idéelle du paysage car elle recouvre une grande variété d'interactions matérielles et immatérielles entre l'homme et son environnement naturel. Ainsi, les Mexica, comme les Tarasques, à partir de leur point de vue, insulaire ou riverain, vont concevoir, utiliser et exploiter cet espace clos au moyen d'embarcations, d'aménagements, d'installations, de routes aquatiques et d'activités économiques, politiques, religieuses ou guerrières. Nous le retrouvons dans un certain nombre de cartes réalisées peu avant ou peu après la Conquête par des érudits indigènes, intimement imprégnés par ce paysage. C'est parce qu'il est encore présent 
aujourd'hui dans le Bassin de Pátzcuaro qu'il participe à la revendication d'une identité culturelle forte. En effet, les pirogues traditionnelles et les filets de pêches "papillon " sont les emblèmes du lac de Pátzcuaro. On en retrouve la représentation dans les sources iconographiques du XVI ${ }^{e}$ siècle, dans le cinéma d'or mexicain, sur les affiches touristiques et même sur les billets de banque nationaux! Ceci démontre que Mexicas et Tarasques s'appuient sur des cultures lacustres qui se définissent par la combinaison d'expériences culturelles, de coutumes, de systèmes cognitifs et de produits issus de la culture matérielle, où la navigation et ses installations associées jouent un rôle central. Les concepts de paysage culturel lacustre et de culture lacustre forment de nouveaux prismes à adopter lorsque, à l'avenir, nous nous intéresserons à ces civilisations.

\section{La canoa : un instrument de transport caractéristique}

8 Comme nous venons de le mentionner, ce travail est basé sur un corpus iconographique qui s'est révélé riche et plus que prometteur. Nous avons étudié vingt et un documents tels que des Codex, des cartes, des fresques, majoritairement datés du XVI ${ }^{\mathrm{e}}$ siècle et sur lesquels sont représentés des embarcations. Cent quarante-neuf ont été identifiés et cent trois ont été classés dans une typologie inédite révélant cinq types d'embarcations différentes. Le premier type d'embarcation plutôt primitif se compose de radeaux, ce qui, dans un environnement marécageux comme Tenochtitlan, semble adapté à l'accès aux matières premières. Á ce jour, aucun reste archéologique de ce type n'a été découvert. Les quatre types suivants sont tous des pirogues monoxyles ou canoa, c'està-dire réalisées à partir d'un seul tronc d'arbre évidé. Les données archéologiques sont également peu nombreuses dans le Bassin de Mexico. Elles se limitent aux miniatures découvertes sur le site du Templo Mayor et de Tlatelolco, auxquels s'ajoute la canoa exposée dans la salle Mexica du Musée national d'anthropologie et d'histoire de Mexico. Toutefois, dans le Bassin de Pátzcuaro, de telles embarcations continuent de naviguer. De plus, nous avons eu le privilège de rencontrer les deux derniers constructeurs de pirogues de la communauté de Comachuén. Leur technique reste inchangée bien qu'ils utilisent aujourd'hui une tronçonneuse, plus performante, pour abattre l'arbre et tabler le tronc. L'évidage lui se fait à la hache et à l'herminette. Bien que diachronique, l'étude comparative de leurs témoignages et de ceux des premiers Européens nous permet d'avancer l'hypothèse selon laquelle il existait différentes tailles de canoa, en fonction de leurs usages et de leurs propriétaires. En effet, Diaz del Castillo précise que la pirogue d'un pêcheur différait de celle transportant l'empereur. Ceci étant confirmé par les photographies des archives du CREFAL et des témoignages ethnographiques enregistrés à Pátzcuaro.

Ainsi, d'après les rares données archéologiques, les sources ethnohistoriques et iconographiques du $\mathrm{XVI}^{\mathrm{e}}$ siècle ainsi que les photographies du début du $\mathrm{XX}^{\mathrm{e}}$ siècle, il existait sur ces lacs de véritables flottes de canoas, mais l'usage qui nous a le plus intéressé reste celui du transport de marchandises. Il permet de répondre à des questions d'ordre technique comme celle, centrale, de la capacité de charge. Grâce au Codex Mendoza, nous savons que ces embarcations transportaient des matériaux de construction comme des roseaux, de la terre, du bois, du sable ou encore de la pierre. D'autres sources comme Cortès mentionnent également le transport des denrées alimentaires ou encore du tribut en provenance des confins de chaque empire. S'intéresser aux caractéristiques techniques de ces embarcations a favorisé notre 
appréhension de l'exploitation de ces deux paysages culturels lacustres. En effet, dans le milieu fortement urbanisé de Tenochtitlan, de nombreux archéologues se sont interrogés sur le transport des grands monolithes sculptés découverts dans l'enceinte du Recinto Sagrado du Templo Mayor. Ces quatre sculptures monumentales ayant une envergure moyenne de 3,50 mètres pour une masse variant entre 8 et 25 tonnes ne pouvaient être transportées sur l'île par voie terrestre. Bien qu'ils s'accordent tous sur ce point, aucun n'avait exploré plus loin cette interrogation primordiale. C'est pourquoi ce travail a proposé d'estimer la capacité de charge d'une embarcation à partir des formules de jauges. Bien qu'empiriques, elles permettent d'estimer de manière approximative le volume d'une embarcation. Nous avons évalué qu'une embarcation comme la canoa de la salle Mexica pouvait transporter de lourdes charges approchant une tonne. Amarrée à une ou deux autres pirogues similaires, le transport de ces monolithes semblerait possible. Grâce à une étude des caractéristiques hydrostatiques, rendue rigoureuse et précise à l'aide d'une modélisation 3D de cette embarcation archéologique, il deviendrait possible, dans le futur, de proposer des résultats plus formels. En outre, la question du transport des pondéreux ne pourra être approfondie qu'en prenant en compte la question du chargement et du déchargement. Á l'avenir, si nous orientons nos recherches en ce sens, il nous faudra aborder cette question.

\section{Anthropisation et urbanisation du milieu lacustre}

10 Les paysages culturels lacustres des bassins de Mexico et Pátzcuaro, traversés par de véritables flottes de canoas, nous ont menés à nous intéresser aux routes qu'elles empruntent. Lacustres ou terrestres, les routes sont les seules preuves tangibles d'une organisation structurelle de ces espaces lacustres. En tant qu'elles reflètent une modalité d'adaptation culturelle, ces routes comprennent à la fois leurs propres avantages et limites. Grâce aux nombreuses recherches effectuées sur les routes en milieu terrestre, mais aussi à la définition de paysage culturel propre à ces deux civilisations, nous savons que le tracé des routes préhispaniques répond à une logique propre. Leur utilité première n'est pas d'ordre économique, mais vise à desservir au mieux l'ensemble du territoire et des sites occupés. C'est grâce à cette conception idéelle du paysage que routes aquatiques et terrestres constituent la continuité l'une de l'autre. Ainsi, nous avons proposé de réutiliser la typologie existante de routes formelles et informelles, deux types de routes terrestres mésoaméricaines adaptées également à nos deux contextes lacustres.

11 Les routes formelles présentent trois caractéristiques: un alignement marqué, une largeur définissable et un manque de déviation. Ces routes sont les preuves matérielles d'une volonté d'organisation et de planification spatiale qui entraîne la réalisation de nombreux travaux d'ingénierie contrôlés et planifiés par un appareil politique centralisé. Facilement identifiables dans le contexte urbain de Tenochtitlan, ces routes sont les grandes Acequias, véritables autoroutes aquatiques qui traversaient Tenochtitlan en desservant les centres économiques, politiques et religieux les plus importants de la capitale Mexica. Nous mentionnerons l'Acequia Real ou encore l'Acequia de la Merced qui ont toutes deux fait l'objet de rapports de fouilles. Dans le Bassin de Pátzcuaro, elles peuvent être identifiées en reliant les trois centres de Tzintzuntzan, Pátzcuaro et Asajo via l'emplacement des sites riverains et insulaires du lac. 
12 Les routes informelles sont quant à elles des routes de nécessités qui ne présentent aucun type de planification ou d'entretien de la part d'un pouvoir centralisé. Elles se dessinent en fonction des besoins de ses usagers. Elles s'adaptent complètement à la topographie du territoire qu'elles traversent, ce qui peut les rendre impraticables en fonction des saisons. Quel que soit le milieu concerné et d'après les données iconographiques et ethnohistoriques, ces routes se définissent par rapport à trois niveaux : familial, communautaire et seigneurial. La caractérisation du niveau de ces routes informelles est intimement liée à leur proximité d'une route formelle. Dans nos deux contextes lacustres, de tels exemples sont représentés sur des documents iconographiques comme le Plano en Papel de Maguey (vers 1558) ou le Codex Reese (vers 1560), tous deux réalisés par des scribes indigènes.

13 Les routes lacustres, qu'elles soient formelles ou informelles, ont toutes un point de départ et un point d'arrivée intimement liés au changement de milieux aquatique et terrestre. À cet endroit précis, les théoriciens de l'archéologie maritime ont observé le développement d'installations spécifiques à la pratique de la navigation. Ces aménagements et installations matérialisent de manière organisationnelle la continuité qui existe entre les routes aquatiques et terrestres. Ils sont nécessaires pour faciliter la transition des hommes ou des marchandises, mais aussi leur coordination entre deux milieux et deux modes de transports différents. Ils se caractérisent par la présence de marqueurs anthropiques spécifiques qui, une fois identifiés et localisés, pourraient permettre, lors de recherches futures, de délimiter différentes zones d'occupation et d'administration du territoire.

Les zones de transition sont des espaces naturels ou artificiels aménagés pour faciliter un transit rapide dans les échanges sur une zone de faible envergure. Ainsi, dans les bassins de Mexico et Pátzcuaro, nous avons identifié trois zones de transitions: les plages, les embarcadères/débarcadères et les quais. D'un point de vue archéologique, seules les structures artificielles seraient plus facilement identifiables, tel l'embarcadère de l'Acequia Real, celui du Palais de Moctezuma à Mexico ou encore les quais artificiels de l'île de Pacanda dans le lac de Pátzcuaro. Cependant, en raison d'un besoin permanent de maintenance des systèmes de transport, qu'ils soient lacustres ou terrestres, ces zones de transition ne peuvent fonctionner sans l'appui de zones de coordination.

Les zones de coordinations correspondent à des regroupements d'installations dédiées au contrôle et au stockage des moyens de transports, du personnel et des cargaisons. Nous incluons donc les ports, les entrepôts, les douanes, les arsenaux et les ponts. Chacune de ces installations est dépendante des autres pour cristalliser, soit un pôle économique, soit un pôle militaire, indispensables au bon fonctionnement de l'autorité politique en place. Dans le Bassin de Mexico, grâce aux sources ethnohistoriques et archéologiques disponibles à ce jour, onze ports ont été recensés, dont quatre se situaient aux quatre points cardinaux de l'île de Tenochtitlan. Dans le Bassin de Pátzcuaro, ce sont trois ports riverains auxquels s'ajoutaient ceux de chacune des îles du lacs. Ces zones de coordination témoignent d'une administration spécifique visant à s'approprier les différents espaces d'échange via la mise en place de postes de contrôle. Également considérés comme zones de coordination, les sites sacrés qui marquent le paysage culturel lacustre témoignent de la cosmovision et de la culture immatérielle de ces sociétés préhispaniques. Nous mentionnerons les sites lacustres du Pantitlán dans 
le Bassin de Mexico et de l'île de la Pacanda dans le Bassin de Pátzcuaro ou encore les sites sacrés sur les montagnes alentours.

\section{Continuité et verticalité}

Afin de comprendre toute l'importance et l'étendue des principes de continuité et de verticalité au centre de la cosmovision préhispanique, nous allons illustrer notre propos à travers deux exemples empruntés au Postclassique récent. Le Canal de la Viga, dans le bassin de Mexico, et la Route Royale, dans le bassin de Pátzcuaro, sont emblématiques et connues par l'ensemble des sources disponibles. Elles reliaient entre eux les principaux sites insulaires, riverains et terrestres.

Le Canal de la Viga dans le Bassin de Mexico formait une route lacustre qui nécessitait plus de huit heures de navigation depuis le port de Chalco jusqu'au cœur de Tenochtitlan. Son utilisation a été attestée par les Conquistador. Il a été partiellement navigable jusque dans les années 1940-1950! Il s'agissait de l'artère principale d'approvisionnement de la capitale en denrées alimentaires produites dans les lacs d'eau douce du sud du Bassin. Chalco était une zone de coordination principale car elle réceptionnait l'ensemble des tributs ou objets de luxe en provenance des confins de l'empire Mexica, destinés à la capitale et à ses élites. Ce port était stratégique. Il constituait le terminus des routes terrestres traversant la barrière montagneuse du Bassin de Mexico et le point de départ de la route du Canal de la Viga. Le tracé passait par huit sites stratégiques qui participaient à la hiérarchisation des espaces navigables avant de pénétrer au cœur de la capitale.

Identifiée archéologiquement, la Route Royale reliait les sites de la côte pacifique via la Sierra Purépecha, en passant par le village de Comachuén, au lac de Pátzcuaro et aux îles comme celle de Janitzio. D'un point de vue ethnographique, ce tronçon de route était principalement emprunté pour approvisionner la zone lacustre en canoas, au moins depuis le XVI ${ }^{\mathrm{e}}$ siècle. La communauté de Comachuén, située dans les montagnes environnantes du Bassin, est connue pour fabriquer des pirogues de qualité commandées par les pêcheurs et les insulaires de la zone lacustre. Elle permettait aussi les échanges commerciaux de produits de première nécessité comme le bois de cuisine (la leña) contre du poisson ou des canards. Cette route a été utilisée jusque dans les années 1980, avant la construction d'une route goudronnée faisant passer le temps de déplacement vers le lac de cinq heures de marche à trente-cinq minutes en voiture.

19 Il existe donc au cœur de ces deux paysages, et ce jusqu'au début du XIX ${ }^{\mathrm{e}}$ siècle, une continuité et une verticalité qui s'articulent autour des zones portuaires qui favorisent l'intégration des territoires en altitude et lointain, au cœur de ces deux Bassins. Ceci serait donc un point de départ en vue de l'étude d'une archéologie des territoires comme cela a été proposé par Benoît Bérard, à partir de son concept de merritoire dans l'espace caribéen.

20 Ainsi, nous espérons avoir démontré, à partir d'une étude analytique et systématique d'un corpus de données éclectiques, autour de l'exploitation d'un mode de transport aquatique, que la navigation comme trait culturel forme une thématique pleine d'avenir dans ces deux régions et au-delà. Le présent travail a permis de délimiter le cadre d'un nouveau champ de recherche. Riche et inexploré, il s'étend bien plus loin que ce que nous avions tous estimé au début de ces recherches. 
21 Je vous remercie donc à nouveau de votre soutien à travers cette aventure extraordinaire consacrée à la recherche de ces navigateurs insoupçonnés du Nouveau Monde.

\section{NOTES}

1. https://www.youtube.com/watch?v=fH7YsA31tb8

\section{RÉSUMÉS}

Dans une aire culturelle où la géographie conspire contre la fluidité des échanges, les sociétés mésoaméricaines ont su trouver des réponses techniques adaptées à leurs besoins. À une époque où l'acheminement de marchandises et dse biens s'effectue principalement à dos d'homme, certaines civilisations vont se tourner vers un milieu aquatique mythique : les lacs. Ce travail de recherche s'intéresse à la pratique de la navigation lacustre et aux installations spécifiques qui lui sont associées. Ce sujet se positionne dans un cadre pluridisciplinaire, entremêlant archéologie, ethnohistoire et ethnologie. Son objectif premier est de délimiter le cadre d'un nouveau champ de recherche à partir d'une étude analytique et systématique d'un corpus de données éclectiques, autour de l'exploitation d'un mode de transport aquatique. En Mésoamérique, les lacs des Bassins de Mexico et de Pátzcuaro ont été convertis en de véritables centres politiques, économiques et culturels à l'origine de l'émergence de l'Empire mexica et du Royaume tarasque à la période Postclassique (1350-1521). Pourquoi archéologues, historiens et ethnologues continuent-ils d'ignorer la véritable importance de la navigation dans l'étude de la formation et de l'organisation de ces deux civilisations? Dans quelle mesure les données extraites de l'étude des embarcations et des installations lacustres peuvent ouvrir de nouvelles perspectives de recherches? Pour répondre à ces questions, nous avons analysé des données archéologiques (aménagements spécifiques, offrandes, embarcations), des documents ethnohistoriques (chroniques des $\mathrm{XVI}^{\mathrm{e}}$ et $\mathrm{XVIII}{ }^{\mathrm{e}}$ siècles), pictographiques (codex, cartes et peintures murales des $\mathrm{XVI}^{\mathrm{e}}$ et XVIII ${ }^{\mathrm{e}}$ siècles), et iconographiques (photographies du XX $\mathrm{XX}^{\mathrm{e}}$ siècle) ainsi que des recherches ethnologiques (observations de terrain et entretiens). Ce corpus se concentre principalement sur l'époque préhispanique (Postclassique 1325-1521) et coloniale. En utilisant la navigation comme un nouveau prisme d'étude pour traiter du cas mexica et tarasque, nous sommes parvenus à dégager des résultats. Notre principale question était de comprendre ce que l'étude d'un moyen de transport singulier pouvait nous apporter de données nouvelles sur les interactions entre les cultures lacustres et leur environnement. Il apparaît que la navigation a été utilisée par les sociétés mexica et tarasque comme un moyen de transport privilégié pour asseoir leur hégémonie sur ces bassins lacustres aux plans économique, politique et religieux. 
In a cultural area where geography conspires against the fluidity of exchanges, the Mesoamerican societies discovered technical answers adapted to their needs. At a time where the exchange of merchandises and goods relied mainly on human forces, some civilizations turned to a mythical aquatic environment: lakes. This research focuses on the practice of lake navigation and specific facilities that are associated with it. Through the need for a horizontal approach, this research is situated in a multidisciplinary framework that combines archaeology, ethnology and ethnohistory. Its primary objective is to elaborate the framework of a new research field from the analytical and systematic study of a corpus of eclectic data, about the exploitation of a water mean of transport. In Mesoamerica, the greatest concentration of lake systems lies in the Mexican highlands. However, only the Mexico and Pátzcuaro Basin have been converted into real political economic and cultural centres, with the emergence of the Mexica Empire and Tarascan State to the Postclassic period (1350-1521). Why then do archaeologists, ethnologists and historians persist to ignore the true importance of navigation in their study of the formation and organization of these two civilizations? To which extant can we extract from the study of boats and lake installations data that can open new research perspectives? To try to answer these questions, we opted for a transdisciplinary methodology based primarily on maritime archaeology. Although the available data are fragmentary, we propose to extract maximum information in a dialogue with other sources. We cover archaeological data issues (specific facilities, offerings, craft), ethnohistorical documents (chronicles of the sixteenth and eighteenth centuries), pictographic (codex, maps and murals from the sixteenth and eighteenth centuries), and iconographic evidence (photographs of the twentieth century) as well as ethnological research (field observations and interviews). While this entire corpus encompasses a broad time period, it focuses largely on pre-Hispanic times (1325-1521 Post-Classic) and Colonial. Each chapter will be treated according to a multidisciplinary methodology to suit the distinct characteristic aspects of this research. Although our approach to the subject is original and sometimes open to criticism, it helps to visualize our study issues. We therefore caution the reader of the difficulties we will encounter treating a subject that seems to have yet only few scientifically sound data. Using the navigation as a new prism to study the mexica and tarascan cases, we managed to achieve results that certainly deserve to be discussed. Our main concern is to demonstrate the importance that the study of a singular means of transport could enable us to provide new data on the joint interactions that existed between lake Postclassic cultures and their environment. In the mexica and tarascan cases, navigation was used as a preferred means of transportation in order to sit their hegemony over the lake basins on both the economic, political and religious levels.

\section{INDEX}

Mots-clés : histoire des techniques, archéologie, archéologie navale, navigation, bateau, paysage culturel, paysage nautique, lac, hydraulique, transport, pluridisciplinarité

Keywords : history of technology, archeology, naval archeology, nautical landscape, cultural landscape, navigation, ship, lake, hydraulics, multidisciplinarity

Thèmes : Positions de thèse

\section{AUTEUR}

\section{ALEXANDRA BIAR}

Chercheur associée au laboratoire ArchAm UMR8096, je suis spécialisée dans la navigation Préhispanique en Mésoamérique et plus particulièrement dans les eaux intérieures (lacs et 
rivières). Je m'intéresse également aux embarcations monoxyles de tradition indigène, à leur construction et à leur rôle au sein des communautés indigènes actuelles que ce soit au Mexique, au Bélize ou en Guyane française. Ma thèse a été publiée en 2018 aux éditions Archaeopress dans la collection Paris Monographs in American Archaeology (ISBSN :9781784919092). 